\title{
Relações do N, P e K com a fluorescência da clorofila, teores de nutrientes foliares e carboidratos solúveis do caule de Caesalpinia echinata Lam.
}

\author{
Geraldo Rogério Faustini Cuzzuol ${ }^{1,2}$, Emerson Campos Canal ${ }^{1}$, Vinícius Novo Gama ${ }^{1} \mathrm{e}$ \\ Leonardo Valandro Zanetti ${ }^{1}$
}

Recebido: 2.06.2015; aceito: 2.02.2016

\begin{abstract}
Relationships of N, P, and K with the chlorophyll fluorescence, leaf nutrient accumulation, and soluble carbohydrate concentration in the stem of Caesalpinia echinata Lam.). The aim of this study was to evaluate the effect of $\mathrm{N}$, $\mathrm{P}$, and $\mathrm{K}$ in the accumulation of these elements in the leaf and their relations with the chlorophyll a fluorescence parameters and with the concentration of soluble carbohydrates in stems of young plants of Caesalpinia echinata. Eight-month-old plants were grown in polyethylene pots. which received $\mathrm{N}, \mathrm{P}$, and $\mathrm{K}$ in isolated form $\left(\left(\mathrm{NH}_{4}\right)_{2} \mathrm{SO}_{4}, \mathrm{P}_{2} \mathrm{O}_{5}, \mathrm{KCl}\right)$ and the combined form NPK in the ratio 10:10:10 and 04:14:08. The pots were arranged in randomized blocks. The applications were repeated each 90 days during 450 days. The results demonstrated that $C$. echinata is more responsive to N. Plants showed higher leaf $\mathrm{N}$ concentrations and positive correlation with the concentration of chlorophyll a and $\mathrm{b}$. However, the treatments did not influence the potential quantum yield of PSII (FV/FM) and the performance index (PITOTAL). The concentration of sucrose in the stem bark was higher than that of secondary xylem and superior in fertilizers rich in P (P and NPK 04:14:08) suggesting increased demand of substrate for forming the wood in these treatments, which could result in more resistant stems and higher survival of the plants under field conditions.
\end{abstract}

Keywords: Brazilwood, fluorescence, nutrients, soluble carbohydrates

RESUMO - (Relações do N, P e K com a fluorescência da clorofila, teores de nutrientes foliares e carboidratos solúveis do caule de Caesalpinia echinata Lam.). Esse trabalho foi realizado com o objetivo de avaliar o efeito de suplementação de N, P e K no acúmulo foliar desses elementos e suas relações com os parâmetros da fluorescência da clorofila $a$ e com a concentração de carboidratos solúveis em caules de plantas jovens de Caesalpinia echinata Lam. Plantas com oito meses de idade foram cultivadas em vasos que receberam $\mathrm{N}, \mathrm{P}$ e K na forma isolada $\left(\left(\mathrm{NH}_{4}\right)_{2} \mathrm{SO}_{4}, \mathrm{P}_{2} \mathrm{O}_{5}, \mathrm{KCl}\right)$ e combinada na proporção 10:10:10 e 04:14:08, distribuídos em blocos inteiramente casualizados. As aplicações foram repetidas a cada 90 dias durante 450 dias. Os resultados demonstraram que C. echinata é mais responsiva ao N. As plantas apresentaram maiores concentrações de $\mathrm{N}$ foliar e correlação positiva com a concentração das clorofilas $a \mathrm{e} b$. No entanto, os tratamentos não influenciaram no rendimento quântico potencial do FSII (FV/FM) e no índice de desempenho (PITOTAL). A concentração da sacarose na casca do caule foi maior do que do xilema secundário e superior nas adubações fosfatada e em NPK 04:14:08 sugerindo maior demanda de substrato para a formação da madeira nesses tratamentos, o que poderia resultar em caules mais resistentes e maior capacidade de sobrevivência das plantas em condições de campo.

Palavras-chave: carboidratos solúveis, fluorescência, nutrientes, pau-brasil

\section{Introdução}

Estudos recentes têm demonstrado que as arbóreas nativas tropicais respondem à aplicação de $\mathrm{N}$, $\mathrm{Pe} \mathrm{K}$ apresentando maior crescimento e maiores teores de nutrientes foliares. De maneira geral, a fertilização com $\mathrm{N}$ estimulou o crescimento das espécies arbóreas tropicais Semanea inopinata (Cruz et al. 2006) e
Dalbergia nigra (Marques et al. 2006). Em algumas espécies nativas de várzea de florestas tropicais do Panamá, a adubação nitrogenada elevou para $11 \%$ a concentração de $\mathrm{N}$ foliar enquanto na adubação fosfatada o P acumulou em $16 \%$ e o K representou apenas $4 \%$ da concentração foliar sob influência da fertilização fosfatada (Santiago et al. 2011).

1. Universidade Federal do Espírito Santo, Departamento de Ciências Biológicas, Programa de Pós-graduação em Biologia Vegetal, Campus Alaor Queiroz Araújo, 29075-910 Vitória, ES, Brasil

2. Autor para correspondência: gcuzzuol@gmail.com 
$\mathrm{O}$ acúmulo foliar de $\mathrm{N}, \mathrm{P}$ e $\mathrm{K}$ em resposta à fertilização, tem influência nas reações fotossintéticas, nos pigmentos fotossintetizantes (Lambers et al. 2012) e nos fotoassimilados em órgãos dreno, como o caule de arbóreas (Kaakinen et al. 2004).

Já é bem conhecida a relação da fertilização, especialmente a nitrogenada, na concentração da clorofila (Kitajima \& Hogan 2003) e na atividade fotossintética (Coste et al. 2005) em árvores tropicais. Além do $\mathrm{N}$, outros nutrientes como o P e K também têm sido associados com variações da clorofila (Marschner 2012). Porém, seus efeitos na eficiência fotossintética e na partição de fotoassimilados em arbóreas nativas de florestas tropicais ainda não estão claros.

De maneira geral, a maior oferta de nutrientes está relacionada ao aumento da concentração de clorofila, da eficiência fotoquímica $\left(\mathrm{F}_{\mathrm{V}} / \mathrm{F}_{\mathrm{M}}\right)$ e de fotoassimilados em caules na fase de maior atividade celular em arbóreas de regiões temperadas (Iivonen et al. 2001, Kaakinen et al. 2004). Segundo Baker (2008), a luz que chega ao fotossistema II pode seguir três possíveis caminhos que competem entre si: as reações fotoquímicas, a fluorescência da clorofila $a$ e perda por calor. Nesse sentido, a análise da fluorescência pode fornecer importantes informações sobre o estado fisiológico do vegetal bem como seu potencial em relação ao ambiente (Maxuell \& Johson 2000).

Em algumas situações especiais como maior oferta de nutrientes no solo, a força de órgãos fonte (folhas) aumenta culminando no transporte de fotoassimilados para o caule (dreno) de arbóreas (Kozlowski 1992). Nos órgãos drenos, os fotoassimilados podem: a) atender a demanda energética do metabolismo do caule em crescimento, b) ser armazenados na forma de amido (Plomion et al. 2001) ou c) servir de substrato para a formação da madeira (Anttonen et al. 2002). Dessa forma, a análise de carboidratos solúveis em caules de plantas tratadas com fertilizantes pode indicar possível atividade na síntese de polímeros estruturais constituintes da madeira como a celulose e as hemiceluloses, como também, o potencial para o armazenamento de compostos energéticos e o crescimento (Zobel \& Spague 1998).

Das arbóreas nativas tropicais consideradas prioritárias para a recuperação da Mata Atlântica do Brasil a espécie Caesalpinia echinata Lam., o pau-brasil, tem recebido atenção especial nos últimos anos. Amplamente distribuída na costa Atlântica do Brasil no início da colonização, essa espécie chegou à beira da extinção após séculos de exploração devido o grande interesse comercial pelo corante braselina presente no seu cerne e, mais recentemente, na confecção de arcos de instrumentos de corda de reconhecida qualidade internacional (Schimleck et al. 2009). Desse modo, estudos relacionados com a avaliação da fertilização nos parâmetros relacionados à eficiência fotoquímica de plantas de $C$. echinata com o propósito de aumentar seu plantio em áreas de ocorrência natural são relevantes.

Considerando que a razão C:N em plantas declina com o aumento da fertilização (Lambers et al., 2008), pressupomos que a fertilização nitrogenada proporcione melhores atributos fisiológicos incluindo maiores concentrações de $\mathrm{N}$ foliar e pigmentos fotossintetizantes com reflexo na melhor eficiência fotoquímica indicada pelos maiores $\mathrm{PI}_{\mathrm{ABS}}$ e $\mathrm{F}_{\mathrm{V}} / \mathrm{F}_{\mathrm{M}}$. Esse trabalho tem como objetivo avaliar o efeito de suplementação de N, P e K no acúmulo foliar desses elementos e suas relações com os parâmetros da fluorescência da clorofila $a$ e com a concentração de carboidratos solúveis em caules de plantas jovens de Caesalpinia echinata Lam.

\section{Material e métodos}

Material biológico e delineamento experimental Sementes colhidas em maio de 2006 no bosque da Fundação Pau Brasil localizado na cidade de Glória do Goitá, Pernambuco, Brasil ( $\left.8^{\circ} 00^{\prime} 07^{\prime \prime S}, 35^{\circ} 17^{\prime} 34^{\prime \prime W}\right)$ foram germinadas em placas de Petri sobre papel de filtro umedecido com água destilada e isotérmica de $25{ }^{\circ} \mathrm{C}$ sob luz contínua em câmaras de germinação (Mello \& Barbedo, 2007). Plântulas com 10 dias de idade foram transferidas para tubetes de polietileno $200 \mathrm{~mL}$ contendo terra de floresta e mantidas em casa de vegetação sob temperatura média anual de $27 \pm 3{ }^{\circ} \mathrm{C}$, fotoperíodo natural de 11:00 $\pm 1: 00 \mathrm{~h}$. sob radiação fotossinteticamente ativa (RFA) de 250-450 mmol m² $\mathrm{s}^{-1}$. Transcorridos seis meses, em novembro de 2007, foram selecionadas plantas homogêneas tendo em média 3,63 $\pm 0,99$ gramas de massa seca, $8 \pm 2$ folhas, $16,2 \pm 3,3 \mathrm{~cm}$ de comprimento, $3,9 \pm 0,3 \mathrm{~mm}$ de diâmetro. As plantas foram transplantadas para vasos de polietileno com capacidade de $8 \mathrm{~L}$ contendo terra de floresta, sendo cultivadas em casa de vegetação por 60 dias até o início dos tratamentos.

Foram testados os efeitos de N, P e K aplicados isoladamente sendo o $\mathrm{N}$ na forma de sulfato de amônio $\left(\left(\mathrm{NH}_{4}\right)_{2} \mathrm{SO}_{4}\right.$ em 5 g por vaso), o P como superfosfato simples $\left(\mathrm{P}_{2} \mathrm{O}_{5}\right.$ em 5 g por vaso) e o K como cloreto 
de potássio ( $\mathrm{KCl}$ em 1,7 g por vaso). Para a forma combinada foram utilizados NPK 10:10:10 e NPK 04:14:08 (12,0 g por vaso). O tipo de adubação aplicado foi o de cobertura seguido de irrigação. As aplicações foram repetidas a cada 90 dias durante 450 dias. $\mathrm{O}$ experimento foi organizado em blocos casualizados constituído por quatro blocos, seis parcelas e oito repetições, em casa de vegetação, nas mesmas condições ambientais descritas anteriormente. Aos 450 dias após o início do experimento foram analisados os teores de N, P e K foliar, os pigmentos, a fluorescência da clorofila $a$ e a concentração de glicose, frutose e sacarose caulinar.

Concentração de nutrientes - Os foliólulos das folhas medianas completamente expandidas foram lavados em água corrente e passaram por secagem em estufa com ventilação forçada em $40{ }^{\circ} \mathrm{C}$. Os teores dos nutrientes foliares foram determinados segundo metodologia pormenorizadamente descrita por Bataglia et al. (1983). O N total foi determinado pela digestão Microkjeldahl. Para a extração de K e P utilizou-se digestão nítrico-perclórica. A determinação do $\mathrm{P}_{\text {total }}$ foi feita por colorimetria $(725 \mathrm{~nm})$ e o K por espectrofotometria de absorção atômica.

Pigmentos fotossintetizantes - Os pigmentos foram extraídos a partir de foliólulos da região mediana de folhas completamente expandidas, nas mesmas folhas utilizadas para a avaliação de fluorescência aos 450 dias após o início da adubação. Para isso retiraram-se quatro discos foliares de $0,45 \mathrm{~cm}$ de diâmetro com o auxílio de um perfurador de rolha e pesados para a obtenção da massa fresca. Os discos foram imediatamente colocados em tubos de ensaio contendo $5 \mathrm{~mL}$ de dimetil sulfóxido (DMSO) saturado com $\mathrm{CaCO}_{3}$ e incubados no escuro a $60^{\circ} \mathrm{C}$ por 5 horas (Wyka et al. 2007). As leituras das absorbâncias foram realizadas nos comprimentos de onda de 470, 645, $663 \mathrm{~nm}$ e calculadas as concentrações dos pigmentos de acordo com as fórmulas de Lichtenthaler \& Welbum (1983):

Clor $a=\left[\left(12,7 \cdot \mathrm{A}_{663}\right)-\left(2,69 \cdot \mathrm{A}_{645}\right)\right] \cdot \mathrm{V} /(1000 \cdot \mathrm{M})$

Clor $b=\left[\left(22,9 \cdot \mathrm{A}_{645}\right)-\left(4,68 \cdot \mathrm{A}_{663}\right)\right] \cdot \mathrm{V} /(1000 \cdot \mathrm{M})$

Clor $_{\text {total }}=\left[\left(20,2 \cdot \mathrm{A}_{663}\right)-\left(2,69 \cdot \mathrm{A}_{645}\right)\right] \cdot \mathrm{V} /(1000 \cdot \mathrm{M})$

Carot $=[(1000 . \mathrm{A} 470)-(1,82 . \mathrm{Chl} a-85,02 . \mathrm{Chl} b)]$. $\mathrm{V} /(198.1000 . \mathrm{M})$

Onde, Clor e Carot significam clorofila e carotenóides, respectivamente. $\mathrm{A}_{663}, \mathrm{~A}_{645}$ e $\mathrm{A}_{470}$ representam os valores das absorbâncias; $V$ é o volume de DMSO em mL utilizado para a extração e M é a massa fresca dos discos.
Fluorescência da clorofila $a$ - Para a avaliação da fluorescência da Clor $a$ foram utilizados foliólulos da região mediana da primeira folha madura e completamente expandida das plantas 450 dias. As medidas de fluorescência da Clor foram realizadas em folhas intactas, ligadas a planta e adaptadas previamente ao escuro por 30 minutos. A fluorescência transiente da Clor $a$ foi medida às $8: 00 \mathrm{~h}$ por fluorômetro portátil (Handy-PEA, Hansatech Instruments Ltd., King's Lynn Norfolk, UK), induzida pela luz vermelha com pico de $650 \mathrm{~nm}$ de aproximadamente $3.000 \mu \mathrm{mol} \mathrm{m} \mathrm{m}^{-2} \mathrm{~s}^{-1}$. Para análise da fluorescência transiente da Clor $a$ foi utilizado o Teste JIP (Strasser et al. 2004, Tsimilli-Michael \& Strasser 2008), o que permite calcular os fluxos específicos por seção transversal - CS, eficiência ou rendimentos quânticos e índices de performance pormenorizadamente descrito por Yusuf et al. (2010) e Chen et al. (2011).

Carboidratos solúveis - Foram retiradas amostras do caule de duas plantas, por bloco, $5 \mathrm{~cm}$ de distância do colo medindo 0,5-1,0 g de massa fresca e $1 \mathrm{~cm}$ compr. As amostras foram unidas para a obtenção de massa suficiente paras as análises dos carboidratos e da lignina. As amostras foram congeladas em $\mathrm{N}_{2}$ líquido, conservados em ultra freezer $-70^{\circ} \mathrm{C} \mathrm{e}$, posteriormente, foram liofilizadas (Terrone modelo LS 3000). A casca foi separada do xilema e ambos foram triturados, separadamente, em moinho tipo micro-wiley. Os carboidratos solúveis foram extraídos em etanol $80 \%$ a $60{ }^{\circ} \mathrm{C}$ durante $1 \mathrm{~h}$. O material foi filtrado a vácuo e a fase líquida resultante do processo foi diluída até $100 \mathrm{~mL}$ para posterior análise. Os carboidratos solúveis (glicose, frutose e sacarose) foram determinados por cromatografia de troca aniônica de alta eficiência com detector de pulso amperométrico (HPEAC-PAD) através do HPLC Dionex ICS 2500 com uma coluna CarboPac PA1 $(4 \times 250 \mathrm{~mm})$. A concentração para cada monossacarídeo foi obtida de acordo com a cromatografia das amostras com os padrões glicose, frutose e sacarose da Sigma ${ }^{\circledR}$. Todos os procedimentos para extração e análise de carboidratos por HPEACPAD estão pormenorizadamente descritos em American Society for Testing and Materials (2001).

Análise estatística - Para todos os dados foram realizadas análises de variância ANOVA a 5\% de significância e o teste de Tukey utilizando o programa ASSISTAT versão 7.5 beta (Silva, 2009) após ter constatado a normalidade dos valores. Para os parâmetros da fluorescência, a média dos tratamentos foi dividida pela média do controle e sobre os valores 
obtidos foi calculado o logaritmo de base $10\left(\log _{10}\right)$ obtendo, dessa forma, valores relativos ao controle.

\section{Resultados}

$\mathrm{O} \mathrm{N}, \mathrm{P}$ e $\mathrm{K}$ aplicados isoladamente e nas formas combinadas NPK refletiram na maior concentração foliar desses elementos em relação ao controle (tabela 1). Em C. echinata a proporção da concentração de nutrientes seguiu a ordem decrescente $\mathrm{N}>\mathrm{P}>\mathrm{K}$. Diferente do $\mathrm{N}$ e K, cujas concentrações foliares foram similares e independentes da adubação ser isolada ou combinada, a concentração de P foliar foi menor na

Tabela 1. Concentrações de nutrientes nos foliólulos de plantas de Caesalpinia echinata tratadas com diferentes adubos aos 450 dias após o início do experimento. Médias com mesma letra não diferem pelo teste de Tukey a $5 \%$ $(\mathrm{n}=8)$.

Table 1. Concentrations of nutrients in secondary leaflets of Caesalpinia echinata plants treated with different fertilizers at 450 days after the start of the experiment. Means with same letter within each column do not show significant differences by Tukey test at $5 \%(n=8)$.

\begin{tabular}{lccc}
\hline \multirow{2}{*}{ Tratamentos } & $\mathrm{N}$ & $\mathrm{P}$ & $\mathrm{K}$ \\
\cline { 2 - 4 } Controle & \multicolumn{3}{c}{$\mathrm{dag} / \mathrm{kg}$} \\
$\mathrm{N}$ & $1,3 \mathrm{~b}$ & $0,1 \mathrm{c}$ & $0,8 \mathrm{~b}$ \\
$\mathrm{P}$ & $3,1 \mathrm{a}$ & $0,1 \mathrm{c}$ & $0,9 \mathrm{~b}$ \\
$\mathrm{~K}$ & $1,4 \mathrm{~b}$ & $0,5 \mathrm{a}$ & $1,0 \mathrm{~b}$ \\
NPK $(04: 14: 08)$ & $1,5 \mathrm{~b}$ & $0,1 \mathrm{c}$ & $1,5 \mathrm{a}$ \\
NPK $(10: 10: 10)$ & $3,6 \mathrm{a}$ & $0,3 \mathrm{ab}$ & $1,4 \mathrm{a}$ \\
\hline
\end{tabular}

forma NPK 10:10:10 em relação à adubação à base de $\mathrm{P}$ (fosfatada).

As plantas de C. echinata submetidas ao $\mathrm{N}$ aplicado isoladamente e nas formas combinadas de NPK apresentaram maiores concentrações de Clor $a$, Clor $b$, Carot e Clor ${ }_{\text {total }}$ (tabela 2). Esses tratamentos proporcionaram, também, maiores razões de $\mathrm{Chl}_{\text {total }}$ : Carot. A razão Clor $a: b$ não foi influenciada pela adição dos nutrientes.

Diferenças também ocorreram nos parâmetros da fluorescência da Clor $a$ (figura 1). De maneira geral, o $\mathrm{N}$ e P inibiram $\mathrm{F}_{\mathrm{v}} / \mathrm{F}_{\mathrm{M}}$ de $C$. echinata enquanto nenhuma diferença ocorreu na adubação fosfatada e nas formas combinadas de NPK em relação ao controle. Os valores de $\mathrm{F}_{\mathrm{V}}, \mathrm{F}_{\mathrm{M}}$ e $\mathrm{RC} / \mathrm{CS}_{0}$ foram menores na adubação fosfatada e maiores nas adubações nitrogenadas e nas duas formas combinadas de NPK em relação ao controle. Foi observado menor valor de $\mathrm{PI}_{\text {total }}$ de $C$. echinata na adubação fosfatada seguido de melhores resultados na adubação nitrogenada e nas formas combinadas de NPK. Contudo, essa diferença não foi significativa. Os parâmetros $\mathrm{F}_{\mathrm{v}} / \mathrm{F}_{0}, \mathrm{RC} / \mathrm{ABS}$ e $\mathrm{ET}_{0} / \mathrm{TR}_{0}$, que são parâmetros relacionados ao cálculo do $\mathrm{PI}_{\text {total }}$, também não apresentaram diferença significativa (tabela 3 ).

As concentrações dos fotoassimilados caulinares só diferiram quanto à sacarose da casca que apresentou maiores concentrações na adubação fosfatada seguida de NPK 04:14:08 (tabela 4). Independente do compartimento caulinar (xilema secundário e a casca), a proporção de concentração dos açúcares desse órgão dreno seguiu a ordem decrescente sacarose $>$ glicose $>$ frutose sendo que maior

Tabela 2. Concentrações de clorofila $a$ (Clor $a$ ), clorofila $b$ (Clor $b$ ), clorofila total $\left(\right.$ Clor $_{\text {total }}$ ), carotenóides (Carot), razão clorofila $a$ : clorofila $b$ (Clor $a: b)$ e razão clorofila total e carotenóide (Clor ${ }_{\text {total }}$ :Carot) em plantas de Caesalpinia echinata tratadas com diferentes adubos. Médias com mesma letra não mostram diferenças significativas pelo teste de Tukey a $5 \%$ aos 450 dias após o início do experimento $(\mathrm{n}=8)$.

Table 2. Concentrations of chlorophyll a (Chl a), chlorophyll b ( Chl b), total chlorophyll $\left(\mathrm{Chl}_{\text {total }}\right)$, carotenoids (Carot), chlorophyll a: chlorophyll b ratio (Chl a: b), and total chlorophyll and carotenoid ratio (Chltotal:Carot) in Caesalpinia echinata plants treated with different fertilizers. Means with same letter within each column do not show significant differences by Tukey test at $5 \%$ at 450 days after the beginning of the experiment $(n=8)$.

\begin{tabular}{|c|c|c|c|c|c|c|}
\hline \multirow{2}{*}{ Tratamentos } & Clor $a$ & Clor $b$ & Clor $_{\text {total }}$ & Clor $a: b$ & Carot & Clor $_{\text {total }}$ : Carot \\
\hline & \multicolumn{6}{|c|}{$\mathrm{mg} \mathrm{gMF}^{1}$} \\
\hline Controle & $1,2 \mathrm{bc}$ & $1,1 \mathrm{~b}$ & $1,5 \mathrm{c}$ & $1,1 \mathrm{a}$ & $0,7 \mathrm{~b}$ & $2,1 \mathrm{c}$ \\
\hline $\mathrm{N}$ & $1,8 \mathrm{ab}$ & $1,8 \mathrm{a}$ & $3,1 \mathrm{~b}$ & $1,0 \mathrm{a}$ & $1,1 \mathrm{a}$ & $2,8 \mathrm{a}$ \\
\hline $\mathrm{P}$ & $1,0 \mathrm{c}$ & $1,0 \mathrm{~b}$ & $1,7 \mathrm{c}$ & $1,0 \mathrm{a}$ & $0,7 \mathrm{~b}$ & $2,4 \mathrm{bc}$ \\
\hline K & $1,5 \mathrm{bc}$ & $1,5 \mathrm{ab}$ & $2,9 \mathrm{bc}$ & $1,0 \mathrm{a}$ & $0,9 \mathrm{ab}$ & $3,2 \mathrm{bc}$ \\
\hline NPK (04:14:08) & $2,3 \mathrm{a}$ & $2,0 \mathrm{a}$ & $3,8 \mathrm{a}$ & $1,1 \mathrm{a}$ & $1,3 \mathrm{a}$ & $3,0 \mathrm{a}$ \\
\hline NPK (10:10:10) & $2,0 \mathrm{a}$ & $1,8 \mathrm{a}$ & $3,4 \mathrm{ab}$ & $1,1 \mathrm{a}$ & $1,1 \mathrm{a}$ & $3,1 \mathrm{a}$ \\
\hline
\end{tabular}


concentração de sacarose ocorreu na casca chegando a representar $6 \%$ de sua massa seca.

\section{Discussão}

O maior acúmulo de nutrientes foliares nas plantas de $C$. echinata tratadas com fertilizantes confirma observações recentes de que arbóreas tropicais respondem à adição de $\mathrm{N}, \mathrm{P}$ e $\mathrm{K}$ no solo (Santiago et al. 2011). A maior proporção do $\mathrm{N}$ foliar em plantas de C. echinata tratadas com fertilizantes à base de $\mathrm{N}$ (fertilização nitrogenada) também foi observada em algumas arbóreas tropicais da sucessão tardia da flora brasileira como plantas jovens de Swietenia macrophylla adubadas com NPK (Tucci et al. 2007). No entanto, as espécies pioneiras Peltaphon dubin denominada Angico-amarelo (Venturim et al. 1999) e Toone cilata, também conhecida como Cedro australiano (Moretti et al. 2011) mostraram maior exigência ao P. Esses resultados apóiam o conceito de que as pioneiras são mais exigentes ao $\mathrm{P}$ e $\mathrm{K}$ do que as não pioneiras (Duboc, 2005). Nesse sentido, a menor exigência $C$. echinata por esses dois elementos sustenta sua posição como intermediária da sucessão florestal (Mengarda et al. 2009). A menor concentração

Tabela 3. Média dos parâmetros da fluorescência da clorofila $a$, coeficiente de variação $(\mathrm{CV})$ e teste de significância $P<0,05(F)$ aos 450 dias após o início do experimento $(\mathrm{n}=8)$.

Table 3. Average chlorophyll fluorescence parameters, coefficient of variation (CV) and test of significance of $P<0.05(F)$ to 450 days after beginning the experiment $(\mathrm{n}=8)$.

\begin{tabular}{lccc}
\hline Parâmetros da fluorescência & Média & $\mathrm{CV}$ & $F$ \\
\hline $\mathrm{PI}_{\text {total }}$ & 281 & $31 \%$ & $\mathrm{~ns}$ \\
$\mathrm{~F}_{\mathrm{v}} / \mathrm{F}_{0}$ & 396 & $18 \%$ & $\mathrm{~ns}$ \\
$\mathrm{RC} / \mathrm{ABS}$ & 0,39 & $21 \%$ & $\mathrm{~ns}$ \\
$\mathrm{ET}_{0} / \mathrm{TR}_{0}$ & 0,46 & $17 \%$ & $\mathrm{~ns}$ \\
$\mathrm{ABS} / \mathrm{CS}$ & 2,88 & $15 \%$ & $\mathrm{~ns}$ \\
$\mathrm{TR}_{0} / \mathrm{CS}$ & 1,12 & $10 \%$ & $\mathrm{~ns}$ \\
$\mathrm{ET}_{0} / \mathrm{CS}$ & 1048 & $6 \%$ & $*$ \\
$\mathrm{DI}_{0} / \mathrm{CS}$ & 766 & $30 \%$ & $\mathrm{~ns}$ \\
$\mathrm{RC} / \mathrm{CS}$ & 346 & $10 \%$ & $*$ \\
$\mathrm{~F}_{0}$ & 1048 & $15 \%$ & $\mathrm{~ns}$ \\
$\mathrm{~F}_{\mathrm{M}}$ & 3988 & $1 \%$ & $*$ \\
$\mathrm{~F}_{\mathrm{V}}$ & 2941 & $6 \%$ & $*$ \\
$\mathrm{~F}_{\mathrm{v}} / \mathrm{F}_{\mathrm{M}}$ & 0,74 & $5 \%$ & $\mathrm{~ns}$ \\
\hline
\end{tabular}
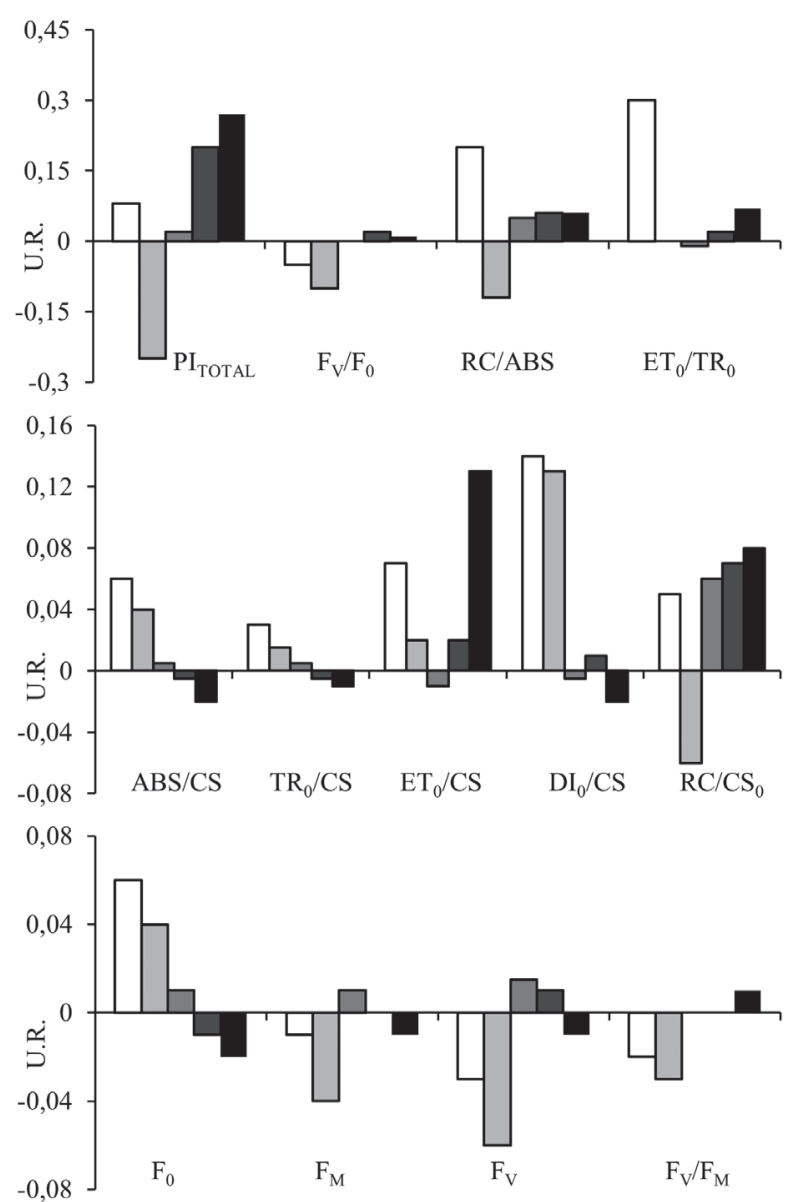

Figura 1. Parâmetros da fluorescência da clorofila $a$ em relação ao controle aos 450 dias após o início do experimento. Fluorescência inicial $\left(\mathrm{F}_{0}\right)$, fluorescência máxima $\left(\mathrm{F}_{\mathrm{M}}\right)$, fluorescência variável $\left(\mathrm{F}_{\mathrm{V}}\right)$, rendimento quântico máximo potencial do FSII $\left(\mathrm{F}_{\mathrm{v}} / \mathrm{F}_{\mathrm{M}}\right)$, absorbância de fótons (ABS/CS), transporte de elétrons ( $\left.\mathrm{ET}_{0} / \mathrm{CS}\right)$, fluxo de energia capturada ( $\left.\mathrm{TR}_{0} / \mathrm{CS}\right)$, dissipação de energia ( $\mathrm{DI}_{0} /$ $\mathrm{CS}$ ), densidade de centros de reação por seção transversal (RC/ $\mathrm{CS}_{0}$ ), índice de desempenho $\left(\mathrm{PI}_{\text {total }}\right)$, rendimento quântico máximo efetivo do FSII $\left(\mathrm{F}_{\mathrm{V}} / \mathrm{F}_{0}\right)$, centros de reação ativos por quantidade de luz absorvida (RC/ABS) e probabilidade de um elétron da $\mathrm{Q}_{\mathrm{A}}$ reduzida mover para a cadeia transportadora de elétrons $\left(\mathrm{ET}_{0} / \mathrm{TR}_{0}\right)$ segundo Chen et al. (2011) e Yusuf et al. (2010). U.R. = Unidade Relativa. $\square$ N, $\square$ P, $\square$ NPK 04:14:08, $\square$ NPK 10:10:10.

Figure 1. Parameters of chlorophyll fluorescence as compared to the control at 450 days after the beginning of experiment. Initial fluorescence $\left(\mathrm{F}_{0}\right)$, maximum fluorescence $\left(\mathrm{F}_{\mathrm{M}}\right)$, variable fluorescence $\left(\mathrm{F}_{\mathrm{V}}\right)$, maximum quantum yield of primary photochemical of PSII $\left(\mathrm{F}_{\mathrm{V}} / \mathrm{F}_{\mathrm{M}}\right)$, photon absorbance $(\mathrm{ABS} / \mathrm{CS})$, electron transport $\left(\mathrm{ET}_{0} /\right.$ $\mathrm{CS}$ ), flow of the captured energy $\left(\mathrm{TR}_{0} / \mathrm{CS}\right.$ ), energy dissipation ( $\mathrm{DI}_{0} /$ $\mathrm{CS})$, density of reaction centers per cross section ( $\mathrm{RC} / \mathrm{CS} 0$ ), the performance index ( $\mathrm{PI}_{\text {total }}$ ), effective maximum quantum yield of PSII $\left(\mathrm{F}_{\mathrm{V}} / \mathrm{F}_{0}\right)$, active reaction centers per amount of light absorbed (RC/ABS), and probability of an electron from reduced $\mathrm{Q}_{\mathrm{A}}$ to move the electron transport chain $\left(\mathrm{ET}_{0} / \mathrm{TR}_{0}\right)$ according to Chen et al. (2011) and Yusuf et al. (2010). U.R. = Relative unit. $\square \mathrm{N}$, $\square \mathrm{P}$, NPK 04:14:08, NPK 10:10:10. 
Tabela 4. Concentração de carboidratos solúveis no xilema secundário e na casca do caule de plantas de Caesalpinia echinata tratadas com diferentes adubos aos 450 dias após o início do experimento. Valores em $\mathrm{mg} \mathrm{g}^{-1} \mathrm{MS}$. Médias com mesma letra não mostram diferenças significativas pelo teste de Tukey a $5 \%(\mathrm{n}=8)$.

Table 4. Concentration of soluble carbohydrates in the secondary xylem and stem bark of Caesalpinia echinata plants at 450 days after the start of the experiment. Values in $\mathrm{mg} \mathrm{g}^{-1} \mathrm{DM}$. Means with same letter within each column do not show significant differences by Tukey test at $5 \%$ at 450 days after the beginning of the experiment $(n=4)$.

\begin{tabular}{lcccccc}
\hline & \multicolumn{5}{c}{ casca } \\
\cline { 2 - 6 } Tratamentos & glicose & frutose & sacarose & glicose & frutose & sacarose \\
\cline { 2 - 6 } & \multicolumn{7}{c}{$\mathrm{mg} \mathrm{g}^{-1} \mathrm{MS}$} \\
\hline Controle & $0,5 \mathrm{a}$ & $0,2 \mathrm{a}$ & $44 \mathrm{c}$ & $0,8 \mathrm{a}$ & $0,3 \mathrm{a}$ & $16 \mathrm{a}$ \\
$\mathrm{N}$ & $0,2 \mathrm{a}$ & $0,1 \mathrm{a}$ & $39 \mathrm{c}$ & $0,3 \mathrm{a}$ & $0,4 \mathrm{a}$ & $11 \mathrm{a}$ \\
$\mathrm{P}$ & $0,4 \mathrm{a}$ & $0,1 \mathrm{a}$ & $62 \mathrm{a}$ & $0,2 \mathrm{a}$ & $0,1 \mathrm{~b}$ & $12 \mathrm{a}$ \\
$\mathrm{K}$ & $0,3 \mathrm{a}$ & $0,1 \mathrm{a}$ & $39 \mathrm{c}$ & $0,2 \mathrm{a}$ & $0,1 \mathrm{a}$ & $0,7 \mathrm{a}$ \\
NPK (04:14:08) & $0,7 \mathrm{a}$ & $0,2 \mathrm{a}$ & $52 \mathrm{~b}$ & $0,2 \mathrm{a}$ & $0,1 \mathrm{a}$ & $12 \mathrm{a}$ \\
NPK (10:10:10) & $0,5 \mathrm{a}$ & $0,1 \mathrm{a}$ & $35 \mathrm{c}$ & $0,2 \mathrm{a}$ & $0,1 \mathrm{a}$ & $12 \mathrm{a}$ \\
\hline
\end{tabular}

de P foliar na forma combinada NPK 10:10:10 em relação à adubação à base de $\mathrm{P}$ (fosfatada) nas plantas de C. echinata também foi constatado em algumas arbóreas tropicais em que a concentração foliar de $\mathrm{P}$ foi menor quando combinado com $\mathrm{N}$ (Lawrence, 2001) sugerindo efeito antagônico do N com o P (Marschner 2012).

Mais uma vez, $C$. echinata mostrou ser mais responsiva à adubação nitrogenada e às formas combinadas de NPK com efeito na concentração dos pigmentos. Resultados semelhantes foram observados em espécies de Bignoniaceae (Kitajima \& Hogan 2003) em que a concentração de Clor $_{\text {total }}$ mostrou correlação positiva com o teor $\mathrm{N}$ foliar nas plantas tratadas com adubação nitrogenada (dados não apresentados). Em C. echinata, o teor de N foliar mostrou, também, correlação significativa com a Clor $_{\text {total }}\left(\mathrm{R}^{2}=0,724, P<0,05\right)$ enquanto o $\mathrm{P}$ não mostrou nenhuma correlação $\left(\mathrm{R}^{2}=0,032\right)$ e o K mostrou menor correlação $\left(\mathrm{R}^{2}=0,499, P<0,05\right)$ em relação ao $\mathrm{N}$ (dados das correlações não apresentados). Essas correlações podem ser interpretadas como reflexo da proporção da concentração dos nutrientes foliares em que o $\mathrm{N}$ foi o elemento mais representativo seguido pelo $\mathrm{P}$ com valores intermediários e pelo $\mathrm{K}$ com os mais baixos valores. A falta de influência das fertilizações na razão da Clor $a: b$ não era esperada uma vez que é bem conhecido que essa proporção aumenta em resposta à maior disponibilidade de nutrientes, especialmente o N (Kitajima \& Hogan 2003). Por outro lado, menor razão Clor $a: b$ foi observado em Fagus sylvatica (espécie de região temperada) sob maior fertilização (Minotta \& Pinzauti 1996). Essa falta de padrão na razão de Clor $a: b$ em relação à maior oferta de nutrientes tem sido atribuído à elevada variabilidade genética interespecífica das arbóreas tropicais (Kitajima \& Hogan 2003).

A falta de diferença de $\mathrm{F}_{\mathrm{V}} / \mathrm{F}_{\mathrm{M}}$ na maior oferta de nutrientes (formas combinadas de NPK) em relação ao controle, também, foi constatada em plantas jovens da arbórea tropical Tabebuia rosea submetidas à maior fertilização (Kitajima \& Hogan, 2003).

Os resultados de $\mathrm{RC} / \mathrm{CS}_{0}$ refletiram no $\mathrm{PI}_{\mathrm{ABS}}$ considerado um indicador da vitalidade da planta permitindo uma análise mais ampla do fotossistema II, em comparação ao $\mathrm{F}_{\mathrm{V}} / \mathrm{F}_{\mathrm{M}}$, relacionando a eficiência de absorção de fótons, a captura de energia de excitação, a densidade de centros de reação ativos e a probabilidade da energia de excitação mover um elétron para além de Qa (Gonçalves et al. 2001). Segundo Hermans et al. (2003), o PI $\mathrm{t}_{\text {total }}$ é importante parâmetro para distinguir o comportamento fotossintético de árvores. Nesse sentido, foi observado menor valor de $\mathrm{PI}_{\text {total }}$ de $C$. echinata na adubação fosfatada seguido dos melhores resultados na adubação nitrogenada e nas formas combinadas de NPK, porém, sem diferença significativa. A falta de diferença significativa nos parâmetros da fluorescência da Clor $a$ nas plantas de C. echinata submetidas à maior disponibilidade de nutrientes também foi constatado por Coste et al. (2005) em 14 espécies de arbóreas tropicais. Esses autores demonstram fraca correlação entre a concentração do $\mathrm{N}$ foliar com a capacidade fotossintética e concluíram que variações na fotossíntese estão mais relacionadas 
com o $\mathrm{N}$ alocado para esta função do que meramente com sua concentração foliar.

Considerando que o $\mathrm{K}$ tem grande participação na fotossíntese e transporte de fotoassimilados no floema (Santiago et al. 2011; Wright et al. 2011) esperava-se encontrar maior concentração de sacarose na casca das plantas de C. echinata adubadas à base de K (fertilização potássica). Todavia, é importante salientar que o transporte ativo da sacarose no floema requer ATP dependente do P em sua estrutura (Marschner 2012). Dessa forma, mesmo que a adubação fosfatada tenha afetado o $\mathrm{PI}_{\text {total }}$ de C. echinata em relação ao controle, esse tratamento proporcionou maior concentração da sacarose na casca do caule de $C$. echinata, sugerindo maior demanda de substrato para a síntese de componentes estruturais da madeira (celulose e as hemiceluloses) conforme postulado por Cuzzuol et al. (2013). Isso poderia resultar em caules mais resistentes e maior sobrevivência das plantas em condições de campo primordial para o reflorestamento com arbóreas nativas tropicais.

Tais resultados sugerem o envolvimento de outros fatores como variações da temperatura e da umidade ambiental que têm profunda influência nas atividades fisiológicas (Lambers et al. 2008). A maior proporção de concentração de sacarose no xilema secundário e na casca de C. echinata confirma informação anterior de que a sacarose é o carboidrato solúvel mais abundante no caule de C. echinata (Geßler et al. 2005).

A falta de diferença significativa de $\mathrm{F}_{\mathrm{v}} / \mathrm{F}_{\mathrm{M}}$ e $\mathrm{PI}_{\text {total }}$ entre os tratamentos demonstra que a maior concentração de sacarose na casca de $C$. echinata na adubação fosfatada é mais dependente do gasto de ATP do que da atividade fotoquímica. De certa forma, essa hipótese é sustentada pela relação da concentração de $\mathrm{P}$ foliar com a sacarose da casca $\left(\mathrm{R}^{2}=0,765, P<0,05\right)$. Quanto ao N e K foliar não se verificou correlação dos teores desses elementos com a concentração da sacarose. A concentração da sacarose na casca das plantas tratadas NPK 04:14:08 inferior à fertilização fosfatada pode ser atribuído à ação antagônica do $\mathrm{N}$ ao P (Marschner 2012) na forma combinada como pode ser constatado pela menor concentração foliar do $\mathrm{P}$ em NPK 04:14:08 em relação à fertilização fosfatada nas plantas de $C$. echinata.

\section{Conclusão}

Confirmamos a hipótese inicial de que $C$. echinata é mais responsiva ao $\mathrm{N}$ indicado pelas maiores concentrações de $\mathrm{N}$ foliar que, por sua vez, mostrou estreita correlação com a concentração de pigmentos fotossintetizantes. Por outro lado, rejeitamos a hipótese de que o $\mathrm{N}$ pudesse melhorar a eficiência fotoquímica baseado na falta de diferença significativa de $\mathrm{F}_{\mathrm{v}} / \mathrm{F}_{\mathrm{M}}$ e $\mathrm{PI}_{\text {total }}$ entre os tratamentos.

\section{Agradecimentos}

O primeiro autor agradece a CAPES pela concessão de bolsa de estudos; a FAPES Processo-39044823/2007 pelo suporte financeiro; ao Eng. Florestal Dr. José Manoel Lúcio Gomes do DCBIO/CCHN/UFES pela orientação no delineamento experimental.

\section{Literatura citada}

American society for testing and materials. 2001. E 1758-01: standard practice for preparation of biomass for compositional analysis. [S.I.].

Anttonen, S., Manninen, A.-M., Saranpã, P., Kainulainen, P., Linder, S. \& Vapaavuori, E. 2002. Effects of longterm nutrient optimization on stem wood chemistry in Picea abies. Trees 16: 386-394.

Baker, N.R. 2008. Chlorophyll fluorescence: a probe of photosynthesis in vivo. Annual Review of Plant Biology 59: 89-113.

Bataglia, O.C., Furlani,A.M.C., Teixeira, J.P.F., Furlani, P.R. \& Gallo, J.R. 1983. Métodos de análise química de plantas. Boletim Técnico 78, Instituto Agronômico de Campinas.

Chen, S., Zhou, F., Yin, C., Strasser, R.J., Yang, C. \& Qiang, S. 2011. Application of fast chlorophyll a fluorescence kinetics to probe action target of 3-acetyl-5-isopropyltetramic acid. Environmental and Experimental Botany 71: 269-279.

Coste, S., Roggy, J., Imbert, P, Born, C., Bonal, D. \& Dreyer, E. 2005. Leaf photosynthetic traits of 14 tropical rain forest species in relation to leaf nitrogen concentration and shade tolerance. Tree Physiology 25:1127-1137.

Cuzzuol, G.R.F., Milanez, C.R.D., Gomes, J.M.L., Labate, C.A., Canal, E.C. 2013. Relationship between $\mathrm{N}, \mathrm{P}$, and $\mathrm{K}$ and the quality and stem structural characteristics of Caesalpinia echinata Lam. plants. Trees 27: 1477-1484.

Cruz, C.A.F., Paiva, H.N. \& Guerrero, C.R.A. 2006. Efeito da adubação nitrogenada na produção de mudas de sete-cascas (Samanea inopinata (Harms) Ducke). Revista Árvore 30: 537-546.

Duboc, E. 2005. Desenvolvimento inicial e nutrição de espécies arbóreas nativas sob fertilização, em plantios de recuperação de áreas de cerrado degradado. Tese de Doutorado, UNESP, Botucatu.

Geßler, A., Duarte, H.M., Franco, A.C., Lüttge, U., Mattos, E.A., Nahm, M., Rodrigues, P.J.E.P., Scarano, F.R. \& Rennenberg, H. 2005. Ecophysiology of selected tree species in different plant communities at the periphery of the Atlantic Forest of SE - Brazil III. Three legume in a semi-deciduous dry forest. Trees 19: 523-530. 
Gonçalves, J.F.C., Marenco, R.A., Vieira, G. 2001. Concentration of photosynthetic pigments and chlorophyll fluorescence of mahogany and tonka bean under two light environments. Revista Brasileira de Fisiologia Vegetal 13:149-157.

Hermans, C., Smeyers, M., Rodrigez, R.M., Eyletters, M., Strasser, R.J.\& Delhaye, J. 2003. Quality assessment of urban trees: A comparative study of physiological characterization, airborne imaging and on site fluorescence monitoring by the OJIP-test. Journal of Plant Physiology 160: 8-90.

Iivonen, S., Rikala, R. \& Vapaavuori, F. 2001. Seasonal root growth of Scots pine seedlings in relation to shoot phenology, carbohydrate status and nutrient supply. Canadian Journal of Forest Research 31: 1569-1578.

Kaakinen, S., Jolkkonen, A., Iivonen, S. \& Vapaavuori, E. 2004. Growth, allocation and tissue chemistry of Picea abies seedlings affected by nutrient supply during the second growing season. Tree Physiology 24: 707-719.

Kitajima, K. \& Hogan, K.P. 2003. Increases of chlorophyll $a / b$ ratios during acclimation of tropical woody seedlings to nitrogen limitation and high light. Plant, Cell and Environment 26: 857-865.

Kozlowski, T.T. 1992. Carbohydrate sources and sinks in woos plants. The Botanical Review 58: 107-222.

Laurence, D. 2001. Nitrogen and phosphorus enhance growth and luxury consumption of four secondary forest tree species in Borneo. Journal of Tropical Ecology 17: 859-869.

Lambers H., Raven, J.A., Shaver, G.R., Smith, S.E. 2008. Plant nutrient-acquisition strategies change with soil age. Trends in Ecology \& Evolution 23: 95-103.

Lichtenthaler, H.K. \& Welburn, A.R. 1983. Determination of total carotenoids and chlorophylls $a$ and $b$ of leaf extracts in different solvents. Biochemical Society Transactions 11:591-592.

Marques, V.B., Paiva, H.N., Gomes, J.M., Neves, J.C.L. \& Bernardino, D.C.S. 2006. Efeito de fontes e doses de nitrogênio sobre o crescimento inicial e qualidade de mudas de jacarandá-da-bahia (Dalbergia nigra (Vell.) Fr. All. Ex Benth.). Revista Árvore 30: 725-735.

Marschner, P. 2012. Mineral nutrition of higher plants. 3 ed., Academic Press, Orlando, pp. 649.

Maxuell, K. \& Johnson, G. N. 2000. Chlorophyll fluorescence - a practical guide. Journal of Experimental Botany 51:659-668.

Mengarda, L.H.G., Souza, R.L.F., Campostrini, E., Reis, F.O., Vendrame, W.A., Cuzzuol, G.R.F.2009. Light as an indicator of ecological succession in brazilwood (Caesalpinia echinata Lam.). Brazilian Journal Plant of Physiology 21: 55-64.

Mello, J.I.O. \& Barbedo, C.J. 2007. Temperatura, luz e substrato para a germinação de sementes de paubrasil (Caesalpinia echinata Lam., Leguminosae Caesalpinioideae). Revista Árvore 31: 645-655.

Minotta, G. \& Pinzauti, S. 1996. Effects of light and soil fertility on growth, leaf chlorophyll content and nutrient use efficiency of beech (Fagus sylvatica L.) seedlings. Forest Ecology and Management 86: 61-71.
Moretti, B.S., Furtini-Neto, A.E., Pinto, S.I.C., Furtini, I.V.\& Magalhães, C.A.S. 2011. Crescimento e nutrição mineral de mudas de cedro australiano (Toona cilata) sob omissão de nutrientes. Cerne, v. 17, n. 4.

Plomion, C., Leprovost, G. \& Stokes, A. 2001. Wood formation in trees 127: 1513-1523.

Santiago, L.S., Kaspari, M., Hedin. I.O., Harms, K.E., Garcia, M.N. \& Corre, M.D. 2011. Potassium, phosphorus or nitrogen limit root allocation, tree growth and litter production in a lowland tropical forest. Ecology 92: 1616-1625.

Schimleck, L.R., Espey, C., Christina, R.M., Evans, R., Taylor, A. \& Muniz, G. 2009. Characterization of the wood quality of pernambuco (Caesalpinia echinata Lam) by measurements of density, extractives content, microfibril angle, stiffness, color, and NIR spectroscopy. Holzforschung 63: 457-463.

Silva, F.A.S. 2009. ASSISTAT.exe: assistência estatística. v. 7.5 beta. Campina Grande: UFCG.

Strasser, R.J., Srivastava, A. \& Tsimilli-Michael, M. 2004. Analysis of the chlorophyll $a$ fluorescence transient. In: G.C. Papageorgiou \& R. Govindjee (org.). Fluorescence transient chlorophyll $a$ fluorescence: a signature of photosynthesis. Spring, Netherlands 14: 321-362.

Tsimilli-Michael, M \& Strasser, R.J. 2008. In vivo assessment of plants vitality: applications in detecting and evaluating the impact of Mycorrhization on host plants. In: A. Varma (org.). Mycorrhiza. Uttar Pradesh, India, pp. 679-703.

Tucci, C.A.F., Souza, P.A, Venturim, N. \& Barros, J.G. 2007. Calagem e adubação para a produção de mudas de mogno (Swietenia macrophylla King). Cerne 13: 299-307.

Venturim, N., Duboc, E., Vale, F.R. \& Davie, A.C. 1999. Adubação mineral do Angico-amarelo (Peltophorum dubium Spreng.) Taub.). Pesquisa Agropecuária Brasileira 34: 441-448.

Wright, S.J., Yavitt, J.B., Wurzburger, N., Turner, B.I., Tanner, E.V.J., Sayer, E.J., Santiago, L.S., Kaspari, M., Hedin. I.O., Harms, K.E., Garcia, M.N. \& Corre, M.D. 2011. Potassium, phosphorus or nitrogen limit root allocation, tree growth and litter production in a lowland tropical forest. Ecology 92: 1616-1625.

Wyka, T., Robakowski, P. \& Zytkowiak, R. 2007. Acclimation of leaves to contrasting irradiance in juvenile trees differing in shade tolerance. Tree Physiology 27: 1293-1306.

Yusuf, M.A., Kumar, D., Rajwanshi, R., Strasser, R.J., Tsimilli-Michael, M., Govindjee \& Sarin, N.B. 2010. Overexpression of y-tocopherol methyl transferase gene in Brassica juncea plants alleviates abiotic stress: physiological and chlorophyll a fluorescence measurements. Biochimica et Biophisica Acta 1797: 1428-1438.

Zobel, B.J. \& Spague, J.R. 1998. Juvenile wood in forest trees. Springer-Verlag, Berlim. 